\title{
Research Square \\ Cloud Computing based E-commerce Management Ontransaction Security Concepts
}

\author{
Jinling Chi ( $\nabla$ qwertyuuii11@sina.com ) \\ Huaibei Vocational \& Technical College \\ Xuefeng Sui \\ Huaibei Vocational \& Technical College \\ Mamoun Alazab \\ Charles Darwin University
}

\section{BalaAnand Muthu}

Adhiyamaan College of Engineering

\section{Research Article}

Keywords: Cloud Computing, E-Commerce, Security, Multi-Criteria Decision-Making

Posted Date: July 20th, 2021

DOI: https://doi.org/10.21203/rs.3.rs-721329/v1

License: (9) This work is licensed under a Creative Commons Attribution 4.0 International License. Read Full License 


\title{
Cloud Computing based E-commerce management ontransaction security concepts
}

\author{
Jinling Chi ${ }^{1 \star}$, Xuefeng Sui ${ }^{2}$, MamounAlazab ${ }^{3}$, BalaAnand Muthu ${ }^{4}$
}

${ }^{\star}, 2$ Huaibei Vocational and Technical College,Huaibei 235000, China.

${ }^{3}$ IT and Environment, Charles Darwin University,Australia.

${ }^{4}$ Department of Computer Science and Engineering, Adhiyamaan College of Engineering, India

*Correspondence: qwertyuuii11@sina.com

\section{Abstract}

The way e-commerce companies do commerce is transformed by cloud computing. Although the usage of cloud technology in e-commerce has grown suddenly, the advantages of cloud services platforms have not yet been exploited, especially for e-commerce applications. For decision-makers, it is vital that the optimized cloud-based computing solution like software as a service (SaaS), platform as a service (PaaS), or infrastructure as a service(IaaS) model is adopted as a multi-criteria decision-making (MCDM) issue and is thus dependent on a trustworthy and secured online shop. A new Cloud computing-based E-Commerce Management (CCECM) approach is provided to tackle the MCDM issue, a multiple-criteria group approach based on an ordering preference strategy by an idealized solution, and relying on the structure for a list of requirements. A small-to-medium firm uses the suggested system to ease the assessment and decision-making of the aspects connected with cloud $e$ commerce with security. The results show that small and moderate-sized ecommerce companies have higher secured and efficient communication. Saas is asuitable alternative for difficulty, dependability, safety and protection, organizational preparedness, and corporate size. At the same time, adaptability and scaling can be enhanced in the choosing of PaaS or IaaS. 
Keywords - Cloud Computing, E-Commerce, Security, Multi-Criteria DecisionMaking

\section{Introduction to e-commerce systems}

E-commerce is a component of e-business, and e-commerce may be defined as the purchase and sale of products and services via a network. The focus of $e$ commerce is on digital technologies, business interactions between companies and individuals [1-2]. E-commerce comprises (it is necessary) a transfer of value since there is trade without digital cash. One of the key elements of ecommerce via business interactions is generating money online, and it is essential to understand that e-commerce technologies have distinct characteristics as described in this article:

1. Ubiquity - The conventional commercial market is an actual site, accessibility to treatment through the flow of documents. Clothing and footwear, for instance, are typically aimed at encouraging people to buy something [3]. The omnipresent meaning of e-commerce may be anywhere. E-Commerce is the globe's driving force behind performance gains [4].

2. Global access -E-commerce enables cross-country commercial interactions to be more accessible and efficient than conventional trade [5]. The possible market scale for e-commerce firms is about equal to the global population's connectivity.

3. Universal guidelines - E-commerce innovations are a unique characteristic and the digital technological benchmark that enables all nations to comply with the technical specification of e-commerce [6-7]. Standards can have a significant impact on the costs of market entrance and the price of the merchandise. The guideline can enable digital workplace easier to exist that can save expenses, and an online commerce portal can also set the price of operating costs at $\$ 12$ per month [8].

4. Richness-An essential component of trade are marketing and promotion. Video, music, animations, banners, signage, etc., can be supplied through ecommerce. It's as sophisticated as TV technology [9]. 
5. Interconnectivity - Electronic trade innovation of the 20th Century is known as interaction to provide two-way contact between companies and customers [10].

6. Concentration of data - Networks have increased substantially as long as the overall volume and level of data for all industries, customers, and companies. The innovation of electronic business reduces the cost of information gathering, storage, connection, and execution [11]. At the very exact moment, information technologies are becoming accurate and timely, and data is more helpful and vital than before.

7. Customization- The E-commerce platform is customizable. The business may be modified to match a name, the hobbies of a person, prior purchase messages, and a particular participant's marketing strategy [12]. The technique can also be customized. Traders might modify the products and services based on consumer preferences or past activity.

In recent years, world commerce and the economy have grown. Trade-in in numerous nations, including Nigerians, became the primary market trend. Furthermore, the tendency of world economic transformation is technological trading [13-14]. The geographic accessibility of both the online and offline is responsible for this. Different forms of trade routes have been established on the internet, promoting commercial, corporate accounts via virtualization [15]. Electronic commerce $(E C)$ has been announced as a prominent and expanding web application by the developing digital technologies, allowing clients, suppliers, and workers to accomplish various purposes and solutions [16].

E-commerce is the operating platform for any economic, governmental, and informational transaction using information and communication technology(ICT). E-commerce is categorized as business to business (B2B), enterprise to the customer (B2C), customer to customer (C2C), customer to business (C2B), intraenterprise e-commerce, and enterprises e-commerce based upon this kind of transactions [17-18]. E-commerce solutions offer business data and enable sales, trade, and purchase (e.g., cost and quantity of the items provided).

In implementing SaaS, PaaS, and IaaS as a general cloud technology pattern, this research analyzes decision-making by an e-commerce manager and its factors. Following this significant objective, the research contributions of this 
articleare: what are the consequences on the deployment of cloud-based $e$ commerce by technology, organizational and environmental conditions? This research expands the studies using a unique CCECM approach, a new 2-fold fuzzy language, within the model to assist the decision-making processes. In the context of the research of the findings under the CCECM paradigm, ecommerce executives have insights into choosing the optimal architecture of cloud services for their purposes.

The rest of the research as follows: section 2 illustrates the background of the e-commerce systems. The proposed Cloud Computing-based E-commerce management (CCECM) approach is designed and implemented in section 3. The software analysis and performance evaluation of the proposed CCECM method is discussed in section 4. Section 5 shows the conclusion and future scope of the proposed CCECM approach.

\section{Background to the e-commerce systems}

An overview can be provided of the principles and cloud variations and the professionals and downsides of e-commerce cloud technology. They analyze the advantages and difficulties of cloud technology and its uses in e-government and e-commerce [19]. The cloud services principles are given based on the business problem and the consequences for the e-commerce market. The classic ecommerce companies and the industry are impacted by cloud services, offer an overview of cloud computingtechnology, emphasize security issues, summarize features, networking security issues, and remedies [20].

It suggested an e-commerce architecture based on cloud technology principles, backgrounds, and trends. The architecture provides an excellent means of tackling the challenge of resource preservation and cost reduction [21]. Furthermore, there is no support for networking security analysis, professional specifications, regulation, and other essential services. Recurrent network architecture is presented for cloud-based computing. The safety procedure of cloud information processing is given to automatically handle B2C networking data through the overlapped connection and modeling parameters [22].

In addition, the overlapped network planning and continuous networking approach were the core of the design, and the combination of several modeling tools provided diverse safety control platforms [23]. Although it is intriguing 
regarding storage space and safety, the suggested structure is inefficient, adequate, and appropriate. It describes the design and features of cloud technology with a specific study of the critical component of e-commerce improvement[24] and emphasizing some of the answers provided by cloud technology to e-commerce, safety, transparency, costs, and so forth. Some of the critical challenges required for reliable cloud-based e-commerce deployment also emerged.

A cloud-based cloud computation fuzzy e-commerce approach (FEA) was suggested [25]. The design is appropriate to solve a few current e-commerce challenges with relatively few developmental factors and optimal regulations on the cloud computer execution environment [26]. The invention is mainly limited by failure to maintain the confidentiality of files and folders by the encrypting component. An integrative conceptual foundation of e-commerce and communication systems is given to promote company growth and technology.

The techniques and methods for execution are not given. A cloud-based ecommerce design has been introduced, which addresses critical e-commerce problems such as connectivity and flexibility [27].A 3G-based, cloud-based, mobile e-commerce system with 2D-Barcode technologies is provided to increase application efficiency. The investigation shows that the technique is interesting concerning ease and security and does not examine diverse information accurate measures [28].

Cloud technology has been established as an alternate computing paradigm where web-based applications consider various customers to obtain a broad range of services, e.g., programming and equipment [29]. A wide array of questions in multiple areas, including cognitive, have been covered by cloud technology.Some scholars are starting to adopt this computer paradigm and transfer their research (programs and information) from regional to cloud settings. One criticalfavored view of clouds is that scholars are not obliged to collect expensive computer infrastructures to perform studies or even install many software items. An ordinary scholar can conduct tests by allocating the critical cloud services [30].

The flexibility in the industry space and significant backing for technology and equipment have made cloud storage one of the most critical technologies for ICT experts. It offers the foundation for on-demand, self-serve computing 
services with all-around access to networking, location-independent pooling of resources, fast flexibility, and an economic model pay per usage [31]. The study has grown from ongoing virtualized, dispersed systems, computing systems, connectivity, and World Wide Web (WWW) and programming solutions.

Cloud processing technologies demand lower implementation costs and more specialists to get better programs. The Cloud computing model plays a crucial part in a financial center. Cloud technology delivers new data exchange and processing methods as new product architecture, networked storage, on-demand accessibility to the outdoors. Under present conditions, the cloud computing architecture draws companies with lower B2B and B2C expenditure and research into e-commerce and mobile technology to achieve high technological levels and high potential implementation.

\section{ProposedCloud Computing based E-commerce management (CCECM) approach}

The suggested architecture would be split into hardware, software, resources managing, servers, and business layers. The hardware level is the lowest layer in the cloud services interface and is the framework's central architecture. It is modeled as an efficient and improved platform for better use of resources. The company data center architecture supports services that guarantee the efficiency, availability, deployment, and fast configuration of the data foundation's assets.

Hardware resources, networks, and storage are brought together as a top software environment with virtualization. The physical hardware pool is enlarged dynamically for continuous power provision to the software e-commerce platforms machines, while the storage is always expandable for extra memory assistance. The software layer is developed for the use of software and virtualization technologies as a communication tool. Many software systems combine to provide a collective experience and the construction and integration of cloud-based apps. 


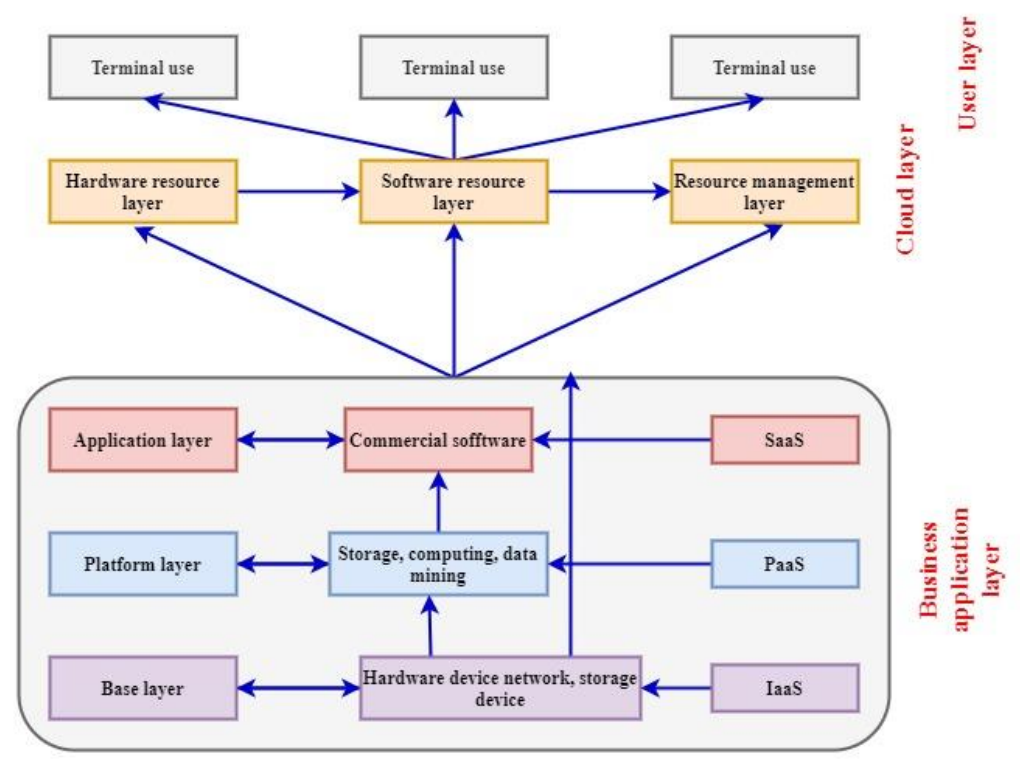

Figure 1. The architecture of the proposed CCECM approach

Figure 1 shows the architecture of the proposed CCECM approach. It has three layers, namely the user layer, cloud layer, and business application layer. The capacity managing component is accountable, in addition to the underlying hardware, for pooling the equipment capabilities and implementing necessary accessibility control lists depending on the accessible underlying hardware. The administration of the latter two levels plays a significant function. The software/hardware capabilities are loosely connected. The system deployment to various virtual machines can be done without interruptions based on the virtualized and planning approach.

This computing model has five main features, three types of services, and four different deployments. Architecture's essential aspects:

Self-service on-demand:It is naturally necessary without human interactions with each network operator. A buyer can organize computer capabilities such as server and systemsstoragewith an attached network.

Complete access to the network: Use standard devices via the internet to enhance client systems, including cellphones, tablets, desktops, and workplaces.

Pooling of resources: The computing capabilities of the suppliers are aggregated to serve different customers utilizing a multi-tenant architecture that effectively allocates and reallocates various digital and physical abilities on demand. Localization senses that the customer typically has no management or 
information about the precise location of the supplied resources and can identify a placement(e.g., countries, states, or datacenter) at a protocol layer. For instance, storing, computation, cognition, and network capacity are instances of commodities.

Quick flexibility: The capacities can be supplied and discharged flexibly to grow outwards quickly and insides, thus sometimes adapting to demand. The capabilities for supply for the customer frequently seem boundless and may be used in any quantity.

Service evaluated: Cloud services regulate and increase resource usage dynamically by using a measuring capacity at a certain level of abstraction suited for the kind of services (e.g., storing, processing, ability, and active consumer details). The use of resources may be monitored, regulated, and communicated, ensuring both the supplier and the customer transparency of the service being used.

\subsection{Service Models}

The servicing layer is separated into three subareas: Services Infrastructure, Services Platform, and Services Software.

\subsubsection{Sofwareas a service (SaaS)}

The opportunity to use the supplier's cloud-based apps is given to the consumer. Apps may be accessed via thin client interaction, a web browser, or a program interface from several mobile terminals. The server does not maintain and operate the fundamental cloud architecture, including networking, computers, software platforms, storing, or different application features, with the potential exception of the restricted user, particular program custom functions.

\subsubsection{Platform as a service (PaaS)}

It is a cloud computing solution that gives clients a framework to develop, operate, and administer programs without creating and managing the architecture generally linked to the development and deployment. In three methods, PaaS can be supplied:

- As a community cloud network providing networking, data centers, processing, operating systems(OS), entity framework (e.g., Java 
runtimes,.NET, latency, implementation, etc.), services to the house, and the consumption request, the customer regulates the data access with negligible additional features and offers the supplier with the platforms, like browsers.

- In the firewalls as a unique facility (software or device)

- As a government platform application used as a service

\subsubsection{Infrastructure as a service (IaaS)}

It is a cloud services type that delivers virtualized internet computer resources. It includes extra help, such as a disc picture collection for a virtual environment, IP numbers, firewalls, virtual localarea network (VLAN), and other packages. Iaas operators request these resources from their vast pools deployed in the cloud services. Businesses can use the website or carriers clouds (virtual private server (VPS) hosting commercial network) provided by IaaS clouds for significant area networking connection.It also offers a standard interface for meaningful online connections, which allows calculation publishing. The fundamental hardware components for the application server are likewise provided by the base layer so that it works in the same way as the target machine.

\subsection{Models for Implementation:}

The proposed model has four cloud architectures: private cloud, public cloud, communication cloud, and hybrid cloud for communication.

\subsubsection{Private cloud}

A solitary firm with several customers provides the network architecture for specific use (e.g., business model). The organization, a person or group, or some combinations of them can own, administer and run it, and it can take place at or out of facilities.

\subsection{2 cloud to the public}

Cloud computing is available to the entire public for unrestricted use. It might be owned, controlled, and administered by or together with a company, academic or governmental organization. It is available at the cloud supplier's facilities.

\subsubsection{Cloud hybridization}


Cloud systems consist of two or more separate (commercial, communal, or public) data centers that stay unique yet are linked by standardized or customized data and applications multi-functioning technologies (e.g., load balance between clouds exploding).

\subsubsection{Communal cloud}

A particular group of customers in businesses that share common interests is provided with a cloud infrastructure for selected usage (e.g., Objectives, safety requirements, policies, and compliance issues).

It can be owned, administered, and maintained by or by a partnership of one or more communal groups like third parties and may be located on or off-site. The layer of commercial application is the procedural code of the platform and frames the expansion made aware. It proposes how corporate objects interface and implement the paths and methods to upgrade and modify business intelligence. Customers may add products, designate shipment and trade activities and provide credit card data to a shopping basket.For illustration, a series of events taking place at the cash register, such as a multi-page form that first demands shipment/shipping documents at the transaction location, following section can appear billing methods. The last section can show the congrats message. The website's enterprise reasoning can also include the process flow.

For this item and specific forms that must be followed, the business regulation of the layout can include the addition of a product from the synopsis section over and over again to increase the amount. Particular data transmission is also included to communicate to the card issuer. Different SaaS solutions can deliver numerous capacity elements that enable companies to operate and control business operations using the cloud platform. They include Sales Monitoring, Product Development, Evaluation, Administration, Management Information Systems, Self-Servers Login Page, Systems Integration, Financial and Accounts Payable, and more. Thus, the proposed structure would ensure that IT personnel on software and hardware are recruited and trained.

Level of Cloud Applications - It is the layer displayed to cloud users. Customers generally access the web-based services supplied by this level and charge penalties for use. 
Cloud Midstream Level - Normally, between windows operating systems and the applications, the cloud gateway level is located. It provides the customer with different features. It assists in developing enterprise systems and facilitates multiple operations, threaded and communication, andmicroservice architecturaldesigns.

\subsection{Workflow of the proposed CCECM approach}

Examples of cloud infrastructure include webpages, server software, and analytics. Middleware programs offer telecommunication by and via and satisfy the demand of a messager to communicate messages from numerous applications. To perform an activity using cloud computing, several apps in many physical places might be "connected."

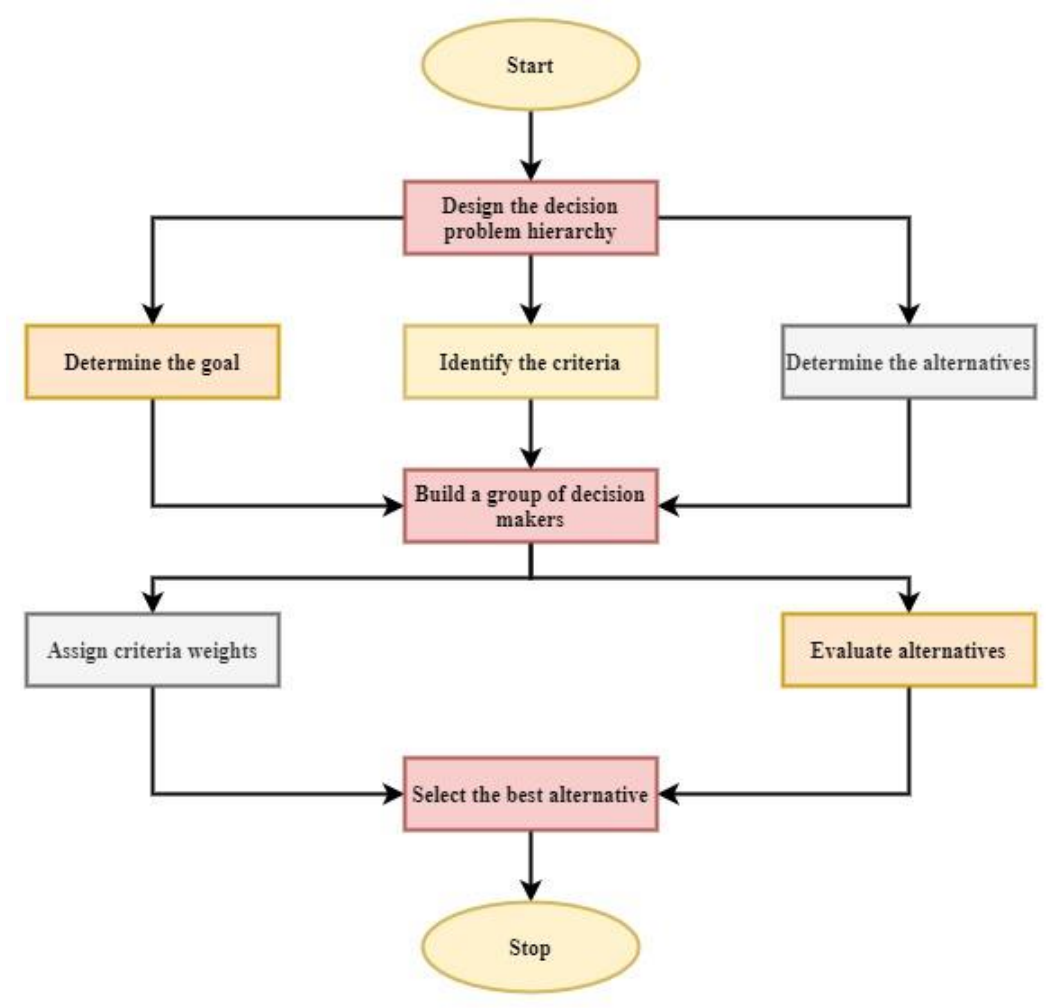

Figure 2. Flowchart of the proposed CCECM approach

Figure 2 shows the flowchart of the proposed CCECM approach. Initially, the decision problem hierarchy is designed. Then the criteria are identified using the goals and alternatives. Based on the requirements, a group decision is made. The decision-makers can help to evaluate the other options and assign criteria weights based on the decision. Finally, the best alternative decision is chosen 
for the problem given. There are the following steps to the suggested selection technique.

\subsubsection{Stage 1}

The primary objective of building the decision-making hierarchy is to choose the most exemplary e-commerce cloud technology architecture. The proposed structure defined the requirements and sub, including three criteria and 12 subcriterion. The options include SaaS, PaaS, and IaaS cloud technology.

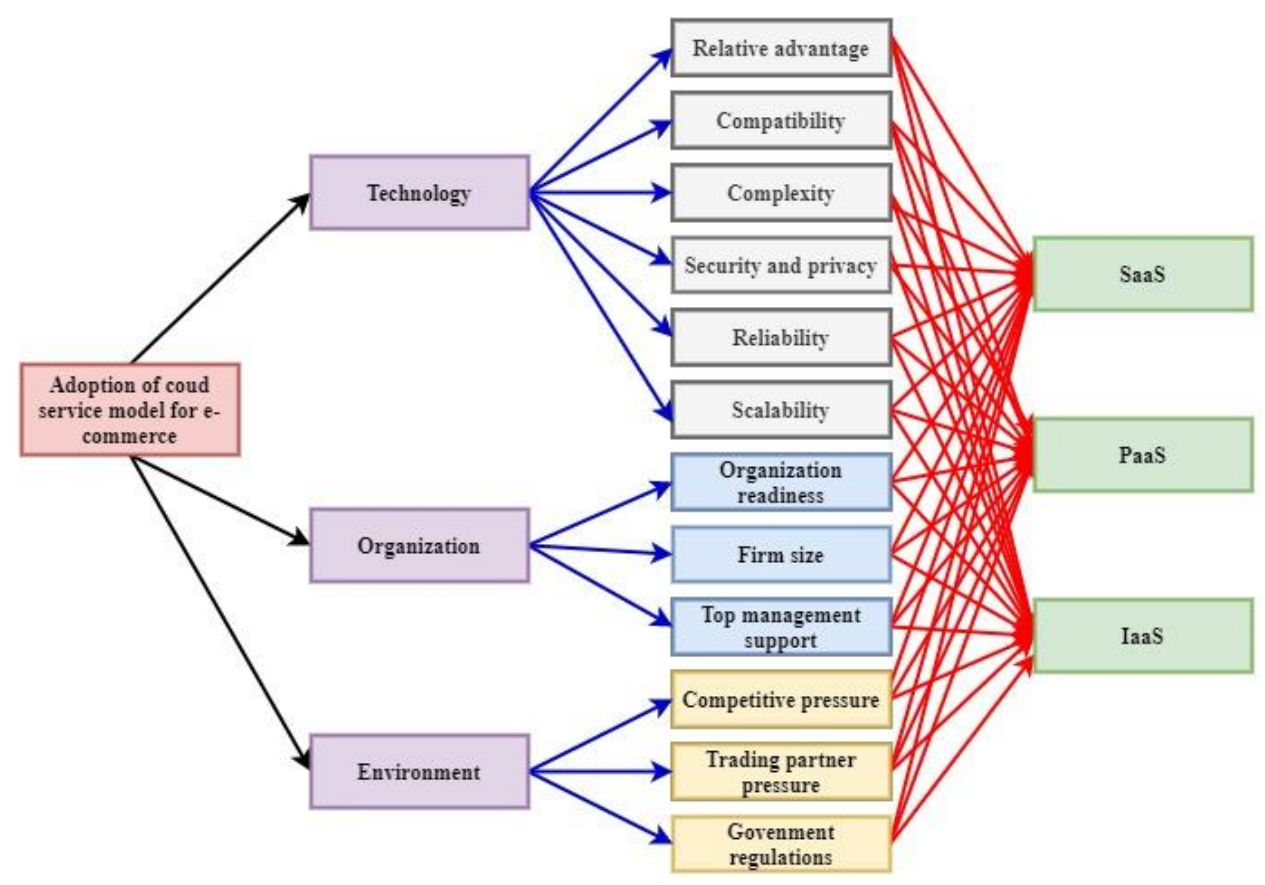

Figure 3. Multi-criteria model of the proposed CCECM approach

Figure 3 shows the multi-criteria model of the proposed CCECM approach. It has three main components such as technology, organization, and environment. Then the modules are subdivided into 12 criteria: relative advantages, compatibility, complexity, security, privacy, reliability, and scalability for the technology module. The organization module consists of organization readiness, firm size, and top management support modules. The environment module has competitive pressure, trading partner pressure, and government regulations.

\subsubsection{Stage 2}

This stage puts together in the firm under examination a party of five executives from various functions. The participants all acknowledged a shared problem (i.e., cloud technology) and tried to achieve a collective conclusion. The 
ultimate judgment was subject to configuration management. Agreement within the collective decision-making indicates that the ending is agreeable to every member truly. With the configuration management, all team members feel equitable opportunities to influence the collective decision and accept it.

The decision-making procedures of the Flexible group typically are based on a rule of agreement and include grading and majority voting rules. The CCECM approach is the best famous and comprehensive way to improve group decisionmaking with configuration management. The CCECMmethod (also known as the CCECM procedure) is intended to achieve multidisciplinary agreement on a viewpoint. It is used the CCECM approach utilizing a survey without having individuals meet one by one to establish the interdisciplinary understanding of the different views.

\subsubsection{Stage 3}

The relevance of the criterion was evaluated and stated in this stage using the fuzzy language factors. For convenience, the included features of theseundefined linguistic parameters are random triangle factors. Linguistic words were also utilized to assess options.

\subsubsection{Stage 4}

The necessary rating was produced by applying the proposed CCECM technique. The ideal approach was selected as the best option with the most significant proximity, the most considerable distance from the anti-ideal approach.

\subsection{Modeling TOPSIS fuzzy group}

The TOPSIS technique is extensively utilized and fuses in many systems; nevertheless, either the language area for lengths is not employed, or the linguistics area is incorrect since the choices' area has been utilized as length domains. In the first example, the criteria are not satisfied as the outcomes are not language-based, and applicability is incorrect.Furthermore, this paper presented a new TOPSIS textual-based CCECM method to help the 2-fold linguistic model when weights and definitions are demonstrated as fuzzy language factors for each measure. Specifications are maintained to produce accurate, light, and easy textual outcomes using appropriate vocabulary and semántics for perceptions and distances. 
Consider that $S=\left\{s_{1}, s_{2}, \cdots, s_{n}\right\}$ is an option set, that $C=\left\{c_{1}, c_{2}, \cdots, c_{n}\right\}$ is the criterion set and that $M=\left\{m_{1}, m_{2}, \cdots, m_{n}\right\}$ is the decision-maker collection. Let $L=\left\{l_{1}, l_{2}, \cdots, l_{n}\right\}$ be the language word to assess the criterion and make $E=$ $\left\{e_{1}, e_{2}, \cdots, e_{n}\right\}$ be a language phrase to evaluate the options. Furthermore, let $E^{1}=$ $\left\{e_{1}, e_{2}, \cdots, e_{n}\right\}$ and $E^{2}=\left\{d_{1}, d_{2}, \cdots, d_{n}\right\}$ be the language word representing the similitude and range between the $l_{p}$ and $l_{r}$ language terms from L.Assume $V_{t}=v_{y}^{t}$ is the weighted matrix in which $v_{y}^{t} \in V$ is the choice of the linguistics value provided to $C_{t} \in C$ by the policymaker $M_{t} \in M$. Further, $A_{t}=a_{x y}^{t}$ is the judgment vector in which $a_{x y}^{t} \in L$ is the preferred language value for $A_{x} \in$ Aconcerning the criterion $C_{t} \in C$, supplied by the judgment $M_{t} \in M$. The significance of every judgment is considered to be the same. The enlarged TOPSIS variant is made up of the following stages:

Phase 1:

$V_{t}=v_{y}^{t}$ is converted into a vector of two-fold language choices $V_{t}=\left(v_{y}^{t}, 0\right)$. The decision vector is denoted as $V_{t}$ and the element of the matrix is denoted as $v_{y}^{t}$.

Phase 2:

The 2-fold grammatical weighted group $V^{T}=\left(\hat{V}_{y}, \hat{\beta}_{y}\right)^{T}$ is expressed in Equation (1)

$\widehat{V}_{y}, \hat{\beta}_{y}=\Delta_{v}\left(\frac{1}{k} \sum_{t=1}^{k} \Delta_{v}^{-1}\left(v_{y}^{t}, 0\right)\right) ; y=1,2, \cdots, n$

The weight of the fuzzy matrix is denoted as $V^{T}$, the element of the matrix is denoted as $\hat{V}_{y}, \hat{\beta}_{y}$. The variation in the weight is denoted as $\Delta_{v}$. The inverse of the variation is denoted $\Delta_{v}{ }^{-1}$. The element of the fuzzy decision matrix is denoted as $v_{y}^{t}$.

Phase 3:

The standardized 2-fold weighted matrix $v^{N}=\left(\hat{\hat{V}}_{y}, \hat{\hat{\beta}}_{y}\right)^{T}$ is expressed in Equation (2)

$\left(\hat{V}_{y}, \hat{\beta}_{y}\right)=\Delta_{v}\left(\frac{\left(\widehat{V}_{y}, \widehat{\beta}_{y}\right)}{\max \left(\left(\widehat{V}_{1}, \widehat{\beta}_{1}\right),\left(\widehat{V}_{2}, \widehat{\beta}_{2}\right), \cdots,\left(\widehat{V}_{n}, \widehat{\beta}_{n}\right)\right)}\right) ; y=1,2, \cdots, n$

The mean of the weight of the fuzzy matrix is denoted as $\left(\hat{V}_{y}, \hat{\hat{\beta}}_{y}\right)$, the mean fuzzy decision matrix element is denoted as $\left(\hat{V}_{y}, \hat{\beta}_{y}\right)$. 
Phase 4:

$A_{t}=a_{x y}^{t}$ is converted into a vector of two-fold language choices $A_{t}=\left(a_{x y}^{t}, 0\right)$. Where $A_{t}$ is the fuzzy decision alternative matrix where the elements of this matrix are denoted as $a_{x y}^{t}$.

Phase 5: The 2-fold linguistics vector of collaborative decisions $A=\left(\hat{a}_{x y}, \widehat{\alpha}_{x y}\right)$ is expressed in Equation (3)

$\left(\hat{r}_{x y}, \hat{\alpha}_{x y}\right)=\Delta_{l}\left(\frac{1}{k} \sum_{t=1}^{k} \Delta_{l}^{-1}\left(a_{x y}^{t}, 0\right)\right) ; y=1,2, \cdots, n, ; x=1,2, \cdots, m$.

The linguistic elements of the fuzzy matrix are denoted as $\left(\hat{r}_{x y}, \hat{\alpha}_{x y}\right)$. The weight variation of the linguistic variable is denoted as $\Delta_{l}$ and the inverse of the linguistic variable is denoted as $\Delta_{l}^{-1}$. The elements of the fuzzy alternative matrix are denoted as $a_{x y}^{t}$. The number of criteria is denoted as $\mathrm{k}$.

Phase 6: The weightingjudgmentvector $\hat{A}=\left(\hat{\bar{a}}_{x y}, \hat{\bar{\alpha}}_{x y}\right)$ is expressed in Equation (4)

$\left(\hat{\bar{r}}_{x y}, \widehat{\bar{\alpha}}_{x y}\right)=\Delta_{l}\left(\Delta_{l}^{-1}\left(\hat{V}_{y}, \hat{\beta}_{y}\right)\right) \Delta_{l}^{-1}\left(\hat{\hat{V}}_{y}, \hat{\hat{\beta}}_{y}\right) ; y=1,2, \cdots, n, ; x=1,2, \cdots, m$.

The mean decision vector weight is denoted as $\left(\hat{\bar{r}}_{x y}, \widehat{\bar{\alpha}}_{x y}\right)$, the linguistic variable is denoted as $\Delta_{l}$, the inverse of the linguistic variable is denoted as $\Delta_{l}{ }^{-1}$. The mean decision matrix is denoted as $\left(\hat{\hat{V}}_{y}, \hat{\hat{\beta}}_{y}\right)$. The weight of the decision matrix is denoted as $\left(\hat{V}_{y}, \hat{\beta}_{y}\right)$.

Phase 7: The positive and negative decisions are expressed in Equations (5) and (6)

$\left(r^{+}, a^{+}\right)=\left\{\left(r_{1}^{+}, a_{1}^{+}\right),\left(r_{2}^{+}, a_{2}^{+}\right), \cdots,\left(r_{n}^{+}, a_{n}^{+}\right)\right\}$

$\left(r^{-}, a^{-}\right)=\left\{\left(r_{1}^{-}, a_{1}^{-}\right),\left(r_{2}^{-}, a_{2}^{-}\right), \cdots,\left(r_{n}^{-}, a_{n}^{-}\right)\right\}$

The positive decision matrix is denoted as $\left(r^{+}, a^{+}\right)$with elements $\left(r_{x}^{+}, a_{x}^{+}\right)$. The negative decision matrix is denoted as $\left(r^{-}, a^{-}\right)$with elements $\left(r_{x}^{-}, a_{x}^{-}\right)$.

Where the elements are denoted in Equations (7) and (8)

$$
\begin{aligned}
& \left(r_{x}^{+}, a_{x}^{+}\right)=\max \left(\left(\hat{r}_{x y}, \hat{\alpha}_{x y}\right) \mid C_{y} \in B\right) \text { or } \min \left(\left(\hat{r}_{x y}, \widehat{\alpha}_{x y}\right) \mid C_{y} \in B^{\prime}\right) \\
& \left(r_{x}^{-}, a_{x}^{-}\right)=\min \left(\left(\hat{r}_{x y}, \hat{\alpha}_{x y}\right) \mid C_{y} \in B\right) \text { or } \max \left(\left(\hat{r}_{x y}, \widehat{\alpha}_{x y}\right) \mid C_{y} \in B^{\prime}\right)
\end{aligned}
$$


$B$ is the defined profit criterion, and wherein $B^{\prime}$ is the defined expense requirements. The relationship vector is denoted as $\hat{r}_{x y}$, the weight of the vector is denoted as $\hat{\alpha}_{x y}$. The criteria for the $y$ user is denoted as $C_{y}$.

Phase 8: The lengths from the excellent response and the unfavorable ideal solutions of each option are computed in Equations (9) and (10)

$$
\begin{aligned}
& \left(\varepsilon_{x}^{+}, \delta_{x}^{+}\right)=\Delta_{l}\left(\frac{1}{n} \Delta_{l}^{-1}\left(m^{2 t}\left(\left(\hat{r}_{x y}, \widehat{\alpha}_{x y}\right),\left(r_{x}^{+}, a_{x}^{+}\right)\right)\right)\right) \\
& \left(\varepsilon_{x}^{-}, \delta_{x}^{-}\right)=\Delta_{l}\left(\frac{1}{n} \Delta_{l}^{-1}\left(m^{2 t}\left(\left(\hat{r}_{x y}, \widehat{\alpha}_{x y}\right),\left(r_{x}^{-}, a_{x}^{-}\right)\right)\right)\right)
\end{aligned}
$$

The linguistic variable is denoted as $\Delta_{l}$ and the inverse of it is expressed as $\Delta_{l}^{-1}$. The relationship vector and weight of the vector is denoted as $\hat{r}_{x y}, \hat{\alpha}_{x y}$. The positive decision is denoted as $r_{x}^{+}, a_{x}^{+}$and the negative decision is denoted as $r_{x}^{-}, a_{x}^{-}$. The fuzzy membership function is denoted as $m^{2 t}$.

Phase 9: Every alternative's proportional proximity to the optimal global solutions is determined in Equation (11)

$\left(\varepsilon_{x}, \delta_{x}\right)=\Delta_{l}\left(\left(\left(\frac{\Delta_{l}^{-1}\left(\varepsilon_{x}^{-}, \delta_{x}^{-}\right)-1}{\left(\Delta_{l}^{-1}\left(\varepsilon_{x}^{-}, \delta_{x}^{-}\right)-1\right)+\left(\Delta_{l}^{-1}\left(\varepsilon_{x}^{+}, \delta_{x}^{+}\right)-1\right)}\right) t^{\prime \prime}\right)+1\right)$

The final decision is denoted as $\left(\varepsilon_{x}, \delta_{x}\right)$, the fuzzy linguistic variable is denoted as $\Delta_{l}$. The inverse of the fuzzy linguistic is denoted as $\Delta_{l}^{-1}$. The negative and positive alternative is denoted as $\left(\varepsilon_{x}^{-}, \delta_{x}^{-}\right)$and $\left(\varepsilon_{x}^{+}, \delta_{x}^{+}\right)$, respectively.

Phase 10: The new variant of proximity establishes alternate solution ranking $\left(\varepsilon_{x}, \delta_{x}\right)$. The most desired alternatives with the most significant linguistic separation are its perception of the range from the anti-ideal resolution.

\section{Software analysis and performance evaluation}

In this research, a section of the review database (http://snap.stanf ord.edu/data/web-amazon.html) is utilized, and offline tests to show various systems' predictive performanceare evaluated. The related documents data, then partition the database into a learning phase, and a testing set by the proportion of 4:1 is created. The simulation is analyzed using the Matlab software with the fuzzy TOPSIS tool. 


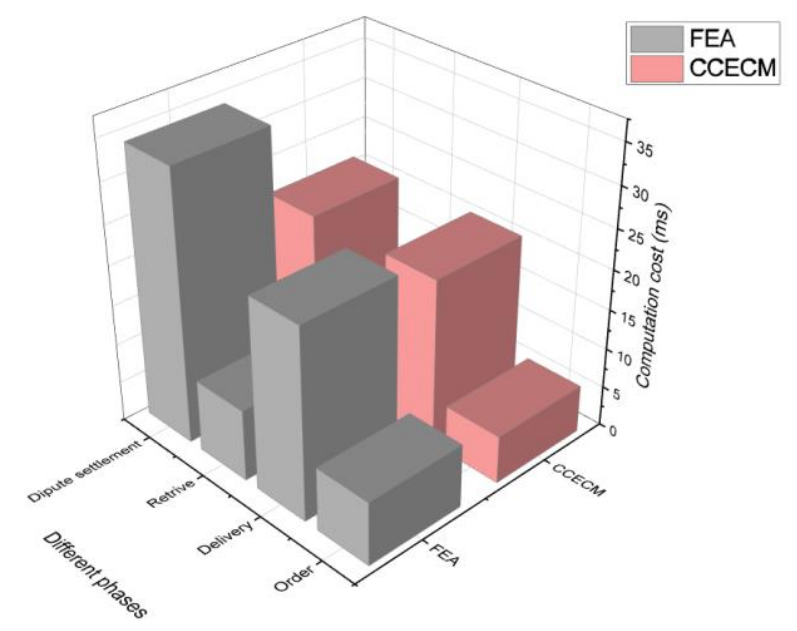

Figure 4(a). Computation cost analysis of the proposed CCECM approach

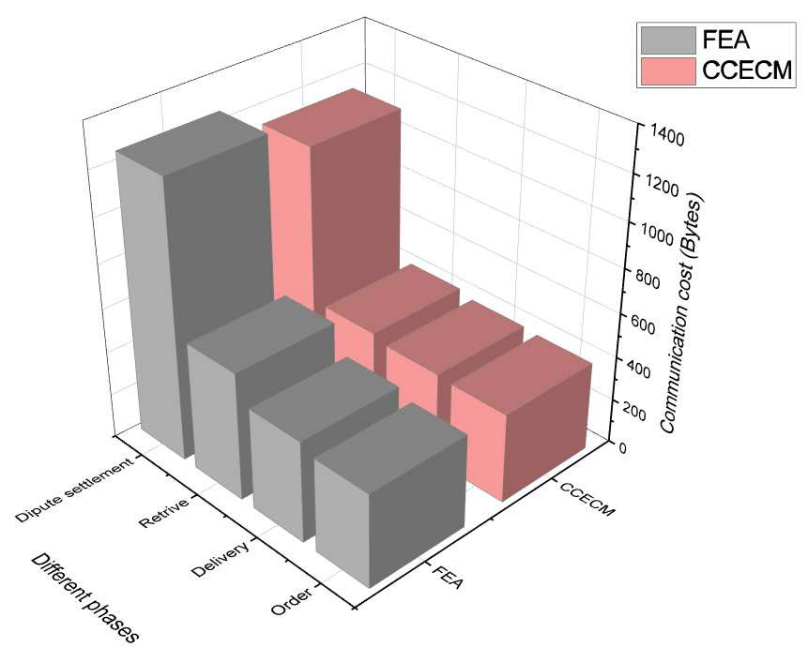

Figure 4(b). Communication cost analysis of the proposed CCECM approach

Figures 4(a) and 4(b) show the computation cost and communication cost analysis of the proposed CCECM approach, respectively. The simulation is carried out by using the amazon consumer dataset. The order, delivery, retrieve, and dispute settlement are analyzed in the simulation for multiple users in different locations. The respective computation cost and communication cost are analyzed and plotted for the proposed CCECM approach compared with the existing FEA model. The results show that the proposed CCECM method has the highest performance of the current model. 
Table 1. Simulation cost analysis of the proposed CCECM approach

\begin{tabular}{|ccccc|}
$\begin{array}{c}\text { Different } \\
\text { phases }\end{array}$ & \multicolumn{2}{c}{ Computation cost (ms) } & \multicolumn{2}{c|}{ Communication cost (Bytes) } \\
\hline Order & 8 & 6 & FEA & CCECM \\
\hline Delivery & 24 & 21 & 424 & 404 \\
\hline Retrive & 9 & 7 & 578 & 412 \\
\hline $\begin{array}{c}\text { Dipute } \\
\text { settlement }\end{array}$ & 34 & 21 & 1254 & 1128 \\
\hline
\end{tabular}

Table 1 shows the simulation cost analysis of the proposed CCECM approach. The simulation analysis is done to deliver, retrieve, and dispute settlement of the different consumers over different time. The computational cost and communication cost of the proposed CCECM approach are analyzed and compared with the existing model. The result indicates that the proposed CCECM method has the highest performance of the current model. The dispute settlement usually takes more time than the others.

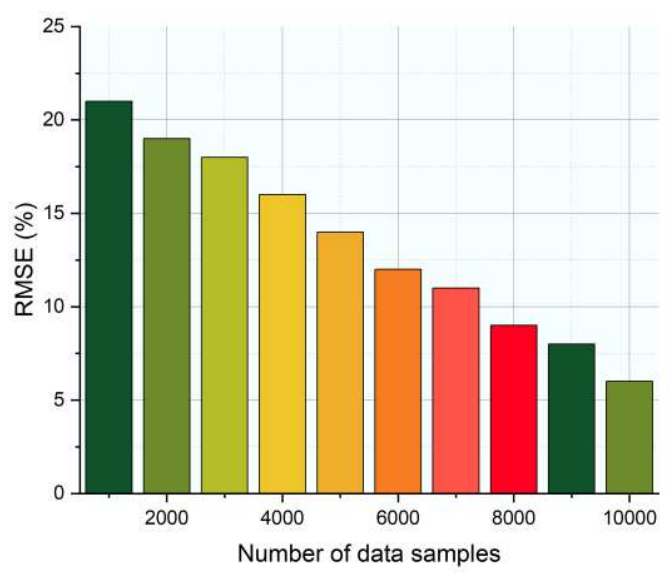

Figure 5(a). RMSE analysis of the proposed CCECM approach 


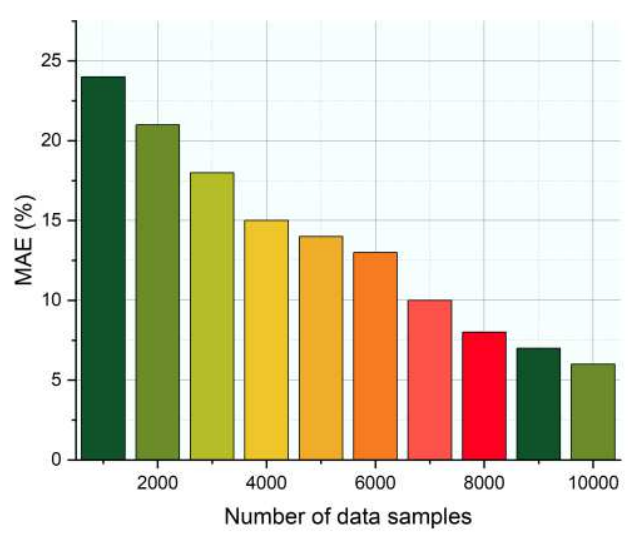

Figure 5(b). MAE analysis of the proposed CCECM approach

Figures 5(a) and 5(b) shows the root mean squared error (RMSE) and mean average error (MAE) analysis of the proposed CCECM approach, respectively. The simulation is carried out by varying the number of datasamples from a minimum of 1000 to a maximum of 10000 with a step size of 1000 . The respective RMSE and MAE of the proposed CCECM approach areanalyzed and plotted in the above figures. As the number of samples increases, the performance increases in terms of a decrease in the error. The result indicates that the proposed CCECM approach has the lowest error for all the situations.

Table 2. Simulation outcome analysis of the proposed CCECM approach

\begin{tabular}{|ccc|}
\hline Method & $\begin{array}{c}\text { Accuracy } \\
(\%)\end{array}$ & Precision (\%) \\
\hline FL & 64 & 67 \\
\hline NN & 72 & 74 \\
\hline RF & 68 & 71 \\
\hline BC & 79 & 81 \\
\hline FEA & 81 & 85 \\
\hline CCECM & 89 & 91 \\
\hline
\end{tabular}


Table 2 shows the simulation outcome analysis of the proposed CCECM approach. The simulation is analyzed for the proposed CCECM method, and the result is compared with the existing models such as fuzzy logic (FL), neural network $(N N)$, random forest (RF), blockchain (BC), andfuzzy e-commerce approach (FEA). The simulation outcomes such as accuracy and precision are analyzed and tabulated in the above table. The results indicate that the proposed CCECM method has the highest performance.

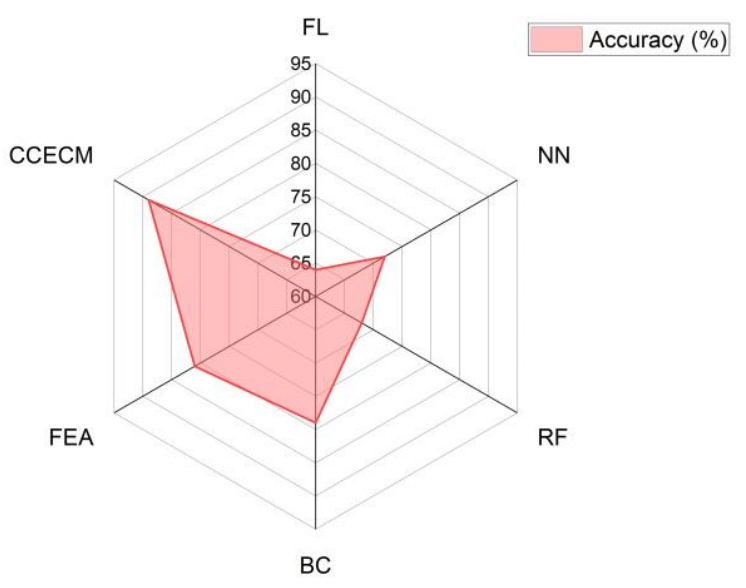

Figure 6(a). Accuracy analysis of the proposed CCECM approach

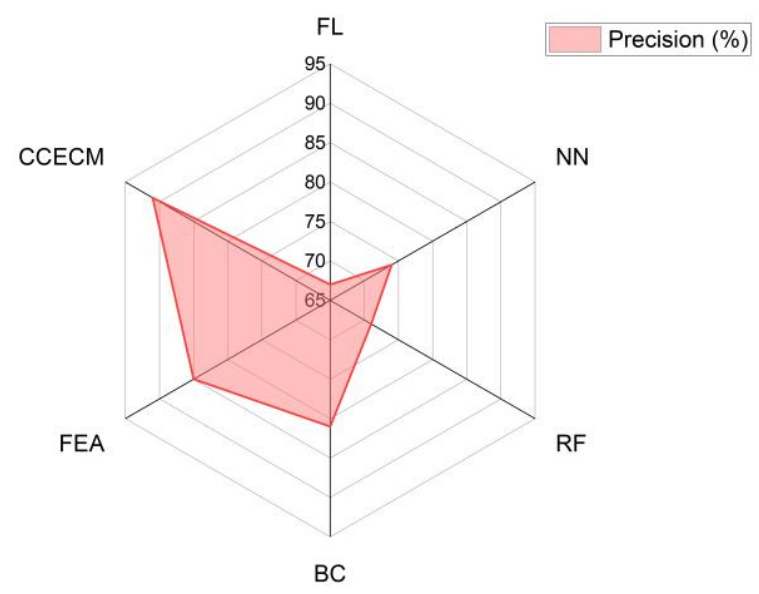

Figure 6(b). Precision analysis of the proposed CCECM approach

Figures 6(a) and 6(b) show the accuracy and precision analysis of the proposed CCECM approach, respectively. The simulation outcomes, such as the precision and accuracy of the proposed CCECM method,areanalyzed and compared with the existing models such as FL, NN, RF, BC, and FEA. The results indicate that 
the proposed CCECM approach has the highest performance of current models in all the scenarios. The proposed CCECM method produces higher marks because of the multi-criteria fuzzy model and cloud computing technology.

The proposed CCECM approach is analyzed, and performance is compared with existing models. The simulation outcomes, such as accuracy, precision, computation cost, communication cost, etc., are evaluated. The results show that the proposed CCECM approach with the help of cloud computing technology produces good results than the existing models in all situations.

\section{Conclusion and findings}

Worldwide competence has a significant role in increasing productivity and effectiveness in e-commerce companies. E-Commerce companies must use the newest technology, such as cloud technology, to enhance their performance and preserve a competitive edge to thrive in this quickly changing industry. The cloud-based e-commerce option, though, is a standard multi-criteria decisionmaking (MCDM) issue. A new Cloud Computing-based E-commerce management (CCECM) approach is developed to cope with this MCDM challenge, which deals with the accurate opinions of policymakers, eliminates the danger of data loss, and promotes a discussion of the results. The study then depends on the CCECM approach to provide an acceptable set of parameters for decision-making. In a smaller to midsized e-commerce company, the recommended technique is applied, and the results show that Saas is the right solution. The effects of this investigation are considerable. Firstly, this research has revealed how public clouds are adopted for small-scale enterprises. Decision-makers can increase decision-making skills depending on the technological characteristics, the organizational needs, and their industry's profitability.Secondly, the proposed policy structure demonstrates that cloud services suppliers aim to enhance their cloud services from technological business administration reasons to satisfy decision makers' criteria in e-commerce businesses. Although numerous elements exist, there are still controversial implications for the three forms of public computational decision-making (IaaS, PaaS, and SaaS).

Furthermore, with the use of two linguistic parameters, the primary contribution of the proposed methodology is the amendment of the TOPSIS technique for solving multi-criteria cooperative decision-making issues. The CCECM approach suggested two foldsare beneficial for any MCDM issue. In 
many other sectors, like e-governments and e-healths, the parameters supplied under the conceptual model are efficient and secured.

\section{Acknowledgement :}

This work was sponsored in part by the Humanities and Social Science Research Project of Universities in Anhui Province (SK2020A0724): Anhui Provincial Teaching Demonstration Class(2180); Anhui Provincial Quality Engineering Major Online Teaching Reform Research Project (2020zdxsjg313).

\section{Ethics Declarations}

Ethical approval

This article does not contain any studies with human participants or animals performed by any of the authors.

\section{Author Statement}

Conception and design of study : Jinling Chi, Xuefeng Sui

Acquisition of data : Xuefeng Sui

Analysis and/or interpretation of data: MamounAlazab, BalaAnand Muthu Conflict of interest

The authors declare that they have no conflict of interest.

\section{References}

1. Seth, S., Johari, R., \& Gupta, K. (2020). Design and Development of ECommerce-Oriented PaaS Application in Cloud Computing Environment. In Applications of Cloud Computing (pp. 131-152). Chapman and Hall/CRC.

2. Thota, C., Manogaran, G., Lopez, D., \&Sundarasekar, R. (2021). Architecture for big data storage in different cloud deployment models. In Research Anthology on Architectures, Frameworks, and Integration Strategies for Distributed and Cloud Computing (pp. 178-208). IGI Global. 
3. Nguyen, T. N., Liu, B. H., Nguyen, N. P., \& Chou, J. T. (2020, June). Cyber Security of Smart Grid: Attacks and Defenses. In ICC 2020-2020 IEEE International Conference on Communications (ICC) (pp. 1-6). IEEE.

4. Jolfaei, A., Jan, M. A., Kant, K., \& Usman, M. (2021). Guest Editorial: Configuration Security for Industrial Automation and Control Systems. IEEE Transactions on Industrial Informatics, 17(6), 4206-4209.

5. Alanezi, A., Abd-El-Atty, B., Kolivand, H., El-Latif, A., Ahmed, A., ElRahiem, A., ... \& S Khalifa, $H$. (2021). Securing digital images through simple permutation-substitution mechanism in cloud-based smart city environment. Security and Communication Networks, 2021.

6. Shakeel, P. M., Baskar, S., Fouad, H., Manogaran, G., Saravanan, V., \& Montenegro-Marin, C. E. (2021). Internet of things forensic data analysis using machine learning to identify roots of data scavenging. Future Generation Computer Systems, 115, 756-768.

7. Hiremath, P. N., Armentrout, J., Vu, S., Nguyen, T. N., Minh, Q. T., \& Phung, P. H. (2019, November). MyWebGuard: toward a user-oriented tool for security and privacy protection on the web. In International Conference on Future Data and Security Engineering (pp. 506-525). Springer, Cham.

8. Billah, M. F. R. M., Saoda, N., Gao, J., \& Campbell, B. (2021, May). BLE Can See: A Reinforcement Learning Approach for RF-based Indoor Occupancy Detection. In Proceedings of the 20th International Conference on Information Processing in Sensor Networks (co-located with CPS-IoT Week 2021) (pp. 132-147).

9. Singh, A. K., Thakur, S., Jolfaei, A., Srivastava, G., Elhoseny, M. D., \& Mohan, A. (2021). Joint encryption and compression-based watermarking technique for security of digital documents. ACM Transactions on Internet Technology (TOIT), 21(1), 1-20.

10. Manogaran, G., Baabdullah, T., Rawat, D. B., \& Shakeel, P. M. (2021). AI Assisted Service Virtualization and Flow Management Framework for 6Genabled Cloud-Software-Defined Network based IoT. IEEE Internet of Things Journal.

11. Galiveeti, S., Lo'aiTawalbeh, M. T., \& Abd El-Latif, A. A. Cybersecurity Analysis: Investigating the Data Integrity and Privacy in AWS and Azure Cloud Platforms. Artificial Intelligence and Blockchain for Future Cybersecurity Applications, 329. 
12. Gao, J., Wang, H., \& Shen, H. (2020, August). Machine Learning Based Workload Prediction in Cloud Computing. In 2020 29th International Conference on Computer Communications and Networks (ICCCN) (pp. 1-9). IEEE.

13. Abdel-Basset, M., Gamal, A., Elhoseny, M., Chakrabortty, R. K., \& Ryan, M. (2021). A Conceptual Hybrid Approach from a Multicriteria Perspective for Sustainable Third-Party Reverse Logistics Provider Identification. Sustainability, 13(9), 4615.

14. Nguyen, T. N., Le, V. V., Chu, S. I., Liu, B. H., \& Hsu, Y. C. (2021). Secure Localization Algorithms Against Localization Attacks in Wireless Sensor Networks. Wireless Personal Communications, 1-26.

15. Manimuthu, A., Dharshini, V., Zografopoulos, I., Priyan, M. K., \& Konstantinou, C. (2021). Contactless Technologies for Smart Cities: Big Data, IoT, and Cloud Infrastructures. SN Computer Science, 2(4), 1-24.

16. Jegadeesan, S., Azees, M., Kumar, P. M., Manogaran, G., Chilamkurti, N., Varatharajan, R., \& Hsu, C. H. (2019). An efficient anonymous mutual authentication technique for providing secure communication in mobile cloud computing for smart city applications. Sustainable Cities and Society, 49, 101522.

17. Abdel-Basset, M., Mohamed, R., Sallam, K., \&Elhoseny, M. (2020). A novel decision-making model for sustainable supply chain finance under uncertainty environment. Journal of Cleaner Production, 269, 122324.

18. Ramasamy, P., Ranganathan, V., Palanisamy, V., \& Kadry, S. (2020). Securing one-time password generation using elliptic-curve cryptography with self-portrait photograph for mobile commerce application. Multimedia Tools and Applications, 79(23), 17081-17099.

19. Shaikh, J. R., Beniwal, R., \& Iliev, G. (2019). Cryptography and optimization-driven support vector neural network to mitigate DoS attacks in E-commerce. In Applications of computing, automation and wireless systems in electrical engineering(pp. 551-561). Springer, Singapore.

20.Sharma, A., \& Banati, H. (2020). Building a Trustworthy Ethical Approach to Cloud Computing. In Data Communication and Networks (pp. 159-181). Springer, Singapore. 
21. Dijesh, P., Babu, S., \& Vijayalakshmi, Y. (2020). Enhancement of ecommerce security through an asymmetric key algorithm. Computer Communications, 153, 125-134.

22.Shanbhog, M. (2021). Cloud Computing Adoption: Influencing Factors on Online Firms. In Innovative Data Communication Technologies and Application (pp. 799-808). Springer, Singapore.

23.Meng, S., He, X., \& Tian, X. (2021). Research on Fintech development issues based on embedded cloud computing and big data analysis. Microprocessors and Microsystems, 83, 103977.

24.Guan, Z., Wang, N., Fan, X., Liu, X., Wu, L., \& Wan, S. (2020). Achieving Secure Search over Encrypted Data for e-Commerce: A Blockchain Approach. ACM Transactions on Internet Technology (TOIT), 21(1), 1-17.

25.Mo, Y. (2019). A data security storage method for IoT under the Hadoop cloud computing platform. International Journal of Wireless Information Networks, 26(3), 152-157.

26.Jiang, W., Wang, Y., Jiang, Y., Chen, J., Xu, Y., \& Tan, L. (2019). Research on mobile internet mobile agent system dynamic trust model for cloud computing. China Communications, 16(7), 174-194.

27.Singh, H., Bhasin, A., \& Kaveri, P. (2019). SECURE: Efficient resource scheduling by swarm in cloud computing. Journal of Discrete Mathematical Sciences and Cryptography, 22(2), 127-137.

28.Guimaraes, T., \& Paranjape, K. (2019). User support as moderator for cloud computing user satisfaction. International Journal of Cloud Computing, 8(2), 150-165.

29.Sehgal, N. K., Bhatt, P. C. P., \& Acken, J. M. (2020). Foundations of cloud computing and information security. In Cloud Computing with Security (pp. 13-48). Springer, Cham.

30.Wangfi, S., Wang, W., \& Tan, Y. (2020). Internet cross-border service model based on $5 G$ environment and cloud computing data platform. Microprocessors and Microsystems, 103520.

31. Siddiqui, Z., Tayan, O., \& Khan, M. K. (2018). Security analysis of smartphone and cloud computing authentication frameworks and protocols. IEEE Access, 6, 34527-34542. 